\title{
ON THE JACOBIAN OF THE KLEIN CURVE
}

\author{
DESPINA T. PRAPAVESSI
}

(Communicated by William Adams)

\begin{abstract}
Its is known that the Jacobian $\mathbf{J}$ of the Klein curve is isogenous to $\mathbf{E}^{3}$ for a certain elliptic curve $\mathbf{E}$. We compute explicit equations for $\mathbf{E}$ and prove that $\mathbf{J}$ is in fact isomorphic to $\mathbf{E}^{3}$. We also identify the subgroup of $\mathbf{J}$ generated by the image of the Weierstrass points of the curve under an Albanese embedding, and we show that it is isomorphic to $\mathbf{Z} / 2 \mathbf{Z} \times(\mathbf{Z} / 7 \mathbf{Z})^{3}$.
\end{abstract}

\section{INTRODUCTION}

Let $\zeta$ be a primitive 7th root of 1 in $\overline{\mathbf{Q}}$, and algebraic closure of $\mathbf{Q}$. Let $k=\mathbf{Q}(\zeta)$, and $\mu_{i}=\zeta^{i}+\zeta^{-i}$. The canonical embedding of the cyclic Fermat quotient curve $t^{7}=s(1-s)^{2}$ in $\mathbf{P}_{\mathbf{Q}}^{2}$ is the Klein curve $\mathbf{X}$, given by the equation

$$
x^{3} y+y^{3} z+z^{3} x=0 .
$$

The curve $\mathbf{X}$ has genus $g=3$ and it is well known that its group of automorphisms is isomorphic to $\mathrm{PSL}_{2}\left(\mathrm{~F}_{7}\right)$ which has order 168 , the maximum for a curve $\mathbf{X}$ of genus 3 . Generators of the group are $[2,5]$

$$
\begin{gathered}
\sigma(x, y, z)=(y, z, x) \text { of order } 3, \\
\tau(x, y, z)=\left(\mu_{1} x+\frac{1}{\mu_{3}} y+z, \frac{1}{\mu_{3}} x+y+\mu_{1} z, x+\mu_{1} y+\frac{1}{\mu_{3}} z\right) \quad \text { of order } 2,
\end{gathered}
$$

and

$$
\varepsilon(x, y, z)=\left(\zeta x, \zeta^{5} y, z\right) \text { of order } 7 .
$$

We will explicitly compute a subgroup of the Jacobian of $\mathbf{X}$ over $K$ isomorphic to $(\mathbf{Z} / 7 \mathbf{Z})^{3}$. The existence of this group was predicted by Greenberg in [3].

We let $\infty_{1}, \infty_{2}, \infty_{3}$ denote the three cusps of $\mathbf{X}$, given by

$$
\infty_{1}=(1,0,0), \quad \infty_{2}=(0,1,0), \quad \infty_{3}=(0,0,1) .
$$

We let $\mathbf{J}_{\mathbf{X}}$ denote the Jacobian of $\mathbf{X}$. To simplify the notation, we also denote by $\sigma, \tau$, and $\varepsilon$ the induced endomorphisms on $\mathbf{J}_{\mathbf{X}}$. Note that by mapping $\zeta$ to $\varepsilon$ we obtain on $\mathbf{J}_{\mathbf{X}}$ complex multiplication by $\mathbf{Z}[\zeta]$. We will identify $\mathbf{Z}[\zeta]$ with its image. We let

$$
\pi=\zeta-1 \in \operatorname{End}\left(\mathbf{J}_{\mathbf{X}}\right) .
$$

Received by the editors March 8, 1993.

1991 Mathematics Subject Classification. Primary 14G05, 14H40; Secondary 14H55. 
The divisors $\infty_{2}-\infty_{1}$ and $\infty_{3}-\infty_{1}$ are both fixed by $\zeta$, and hence have exact order $\pi$. (They are nonprincipal, since the genus of $\mathbf{X}$ is nonzero.)

Recall that $P$ is a Weierstrass point on a curve of genus $g$, if there exists a nonconstant function on the curve with a pole at $P$ of order at most $g$, and no other poles [9].

Let $\mathbf{W}$ denote the set of Weierstrass points on $\mathbf{X}$. We have that

$$
\mathbf{W}=\left\{\theta\left(\infty_{1}\right) \mid \theta \in \operatorname{Aut}(\mathbf{X})\right\}
$$

To show this notice that $\mathbf{W}$ is stable under the action of $\operatorname{Aut}(\mathbf{X})$. Clearly $\infty_{1} \in \mathbf{W}$ since the divisor of $\frac{\mathbf{x}}{y}$ is $\infty_{2}+2 \infty_{3}-3 \infty_{1}$. A direct computation shows that the set on the right side of $(1.1)$ is $\left\{\left(\zeta^{i} \mu_{j}, \zeta^{5 i} \frac{1}{\mu_{3 j}}, 1\right) \mid i=0,1, \ldots, 6, j=\right.$ $1,2,3\} \cup\left\{\infty_{1}, \infty_{2}, \infty_{3}\right\}$. In particular it has order 24 , which is the maximum number of Weierstrass points on a curve of genus 3 .

Let

$$
P=\tau \infty_{1}=\left(\mu_{1}, \frac{1}{\mu_{3}}, 1\right), \quad \mathbf{D}=P+\sigma P-2 \infty_{1} .
$$

We will prove:

Theorem 1. The subgroup $\mathbf{J}_{\mathbf{X}}, \mathbf{w}$ of $\mathbf{J}_{\mathbf{X}}(K)$, generated by the divisor classes of divisors of degree 0 supported on $\mathbf{W}$, is the kernel of $2 \pi^{3}$. In particular the class of $\mathbf{D}$ generates the kernel of $\pi^{3}$ as a $\mathbf{Z}[\zeta]$ module.

Notice that $\mathbf{W}$ is the cuspidal torsion packet [1] of $\mathbf{X}$, studied by Coleman in [2].

Remark. Using similar methods to the ones used here, we have also computed explicitly a subgroup of the Jacobian of the cyclic Fermat quotient curve $t^{13}=$ $s(1-s)^{3}$ over the 13 th cyclotomic field, isomorphic to $\mathbf{Z} / 2 \mathbf{Z} \times(\mathbf{Z} / 13 \mathbf{Z})^{3}$.

We also prove here:

Theorem 2. $\mathbf{J}_{\mathbf{X}}$ is isomorphic over $K$ to the product $\mathbf{E}_{0} \times \mathbf{E}_{1} \times \mathbf{E}_{2}$ of three isomorphic elliptic curves with Weierstrass equations given by

$$
\mathbf{E}_{i}: y^{2}+3 \zeta^{4 i} x y+\zeta^{5 i} y=x^{3}-2 \zeta^{2 i} x-3 \zeta^{3 i}, \quad i=0,1,2 .
$$

Remark. The proof of Theorem 2 can be extended to prove that the Jacobian of the curve $t^{p}=s(1-s)^{r}$, with $p$ prime, $p \equiv 1 \bmod 6$, and $r^{2}+r+1=0 \bmod p$ is isomorphic over the $p$ th cyclotomic field to the product of three isomorphic simple factors. Koblitz and Rohrlich have shown in [6] that the Jacobian of these curves is isogenous to such a product.

In $\S 2$ we compute the equations for the elliptic curves in (1.2) which are the quotient curves of $\mathbf{X}$ under the action of $\varepsilon^{i} \sigma \varepsilon^{-i}$ for $i=0,1$, or 2 . We also compute equations for an isogeny $\phi_{*}$ from $\mathbf{J}_{\mathbf{X}}$ to the product of these three curves. In $\S 3$ we study the action of $\phi_{*}$ on $\mathbf{J}_{\mathbf{X}}\left[\pi^{3}\right]$. Finally in $\S 4$ we prove Theorems 1 and 2.

\section{The isogeny $\phi_{*}$ of $\mathbf{J}_{\mathbf{X}}$ TO The PROduct $\mathbf{E}_{0} \times \mathbf{E}_{1} \times \mathbf{E}_{2}$}

Let $\sigma_{i}=\varepsilon^{i} \sigma \varepsilon^{-i} \in \operatorname{Aut}(\mathbf{X})$. In particular $\sigma_{0}=\sigma$. Since $\sigma_{i}$ has order 3 it generates a cyclic subgroup $\left\langle\sigma_{i}\right\rangle$ of $\operatorname{Aut}_{K}(K(\mathbf{X}))$ of order 3. Let $F_{i}$ denote the fixed field of $\left\langle\sigma_{i}\right\rangle$. Then $K(\mathbf{X})$ is a finite separable extension of $F_{i}$ of degree 3 and the inclusion of fields $F_{i} \subseteq K(\mathbf{X})$ corresponds to a morphism of curves

$$
\phi_{i}: \mathbf{X} \rightarrow \mathbf{E}_{i}
$$


of degree 3. By Hurwitz's theorem $\mathbf{E}_{i}$ has genus 1. Then $\phi_{i}$ induces a unique homomorphism of abelian varieties

$$
\left(\phi_{i}\right)_{*}: \mathbf{J}_{\mathbf{X}} \rightarrow \mathbf{E}_{i}
$$

We have:

Proposition 3. The morphism of abelian varieties $\phi_{*}:=\prod_{i=0}^{2}\left(\phi_{i}\right)_{*}$,

$$
\phi_{*}: \mathbf{J}_{\mathbf{X}} \rightarrow \mathbf{E}_{0} \times \mathbf{E}_{1} \times \mathbf{E}_{2},
$$

is an isogeny.

Proof of Proposition 3. Observe that both $\mathbf{J}_{\mathbf{X}}$ and $\mathbf{E}_{0} \times \mathbf{E}_{1} \times \mathbf{E}_{2}$ have dimension 3. Consider the induced homomorphism of the tangent spaces

$$
d\left(\phi_{*}\right): \mathbf{T}_{\mathbf{J}_{\mathbf{x}}, 0} \rightarrow \mathbf{T}_{\mathbf{E}_{0} \times \mathbf{E}_{1} \times \mathbf{E}_{2,0}},
$$

where $\mathbf{T}_{A, 0}$ denotes the tangent space to the abelian variety $A$, at 0 . It suffices to show that $d \phi_{*}$ is an isomorphism.

Identifying $\mathbf{T}_{\mathbf{J}_{\mathbf{X}}, 0}$ with the vector space of holomorphic differentials on $\mathbf{X}$, we compute a basis $\left\{\omega_{1}, \omega_{2}, \omega_{3}\right\}$ for $\mathbf{T}_{\mathbf{J}_{\mathbf{X}}, 0}$ from the known basis of differentials on the Fermat curve computed in [7, I.7]. We find that

$$
\omega_{1}=\frac{1}{7} \frac{y^{4}}{x^{3}} d\left(\frac{x^{3}}{y^{2}}\right), \quad \omega_{2}=\frac{1}{7} \frac{y^{3}}{x^{2}} d\left(\frac{x^{3}}{y^{2}}\right), \quad \omega_{3}=\frac{1}{7} \frac{y^{3}}{x^{3}} d\left(\frac{x^{3}}{y^{2}}\right) .
$$

Next, notice that $\mathbf{T}_{\mathbf{E}_{0} \times \mathbf{E}_{1} \times \mathbf{E}_{2}, 0}$ is a direct sum of the $\mathbf{T}_{\mathbf{E}_{i}, 0}$ 's for $i=0,1$, or 2 , and a basis for $\mathbf{T}_{\mathbf{E}_{i}, 0}$ is given by the image under $d \phi_{i}$ of any nonzero holomorphic differential which is invariant under the action of $\left\langle\sigma_{i}\right\rangle$. In particular $d \phi_{i}\left(-\omega_{1}+\zeta^{4 i} \omega_{2}-\zeta^{5 i} \omega_{3}\right)$ is a basis for $\mathbf{T}_{\mathbf{E}_{i}, 0}$. Hence we obtain

$$
d \phi_{*}=\left[\begin{array}{rcc}
-1 & -1 & -1 \\
1 & \zeta^{4} & \zeta \\
-1 & -\zeta^{5} & -\zeta^{3}
\end{array}\right]^{-1}
$$

and since the determinant of the above matrix is nonzero, Proposition 3 follows.

Next we will show:

Proposition 4. With notation as above, $\mathbf{E}_{i}$ is the elliptic curve with zero element the point $\mathbf{O}=(0,1,0)$, and Weierstrass equation

$$
y^{2}+3 \zeta^{4 i} x y+\zeta^{5 i} y=x^{3}-2 \zeta^{2 i} x-3 \zeta^{3 i}, \quad i=0,1,2 .
$$

The finite morphism $\phi_{i}: \mathbf{X} \rightarrow \mathbf{E}_{i}$ is given by

$$
\phi_{i}(x, y)=\left(-w_{i}, v_{i}\right)
$$

where

$$
w_{i}=x+\zeta^{6 i} \frac{1}{y}+\zeta^{4 i} \frac{y}{x}, \quad v_{i}=y+\zeta^{6 i} \frac{1}{x}+\zeta^{2 i} \frac{x}{y} .
$$

Furthermore each $\mathbf{E}_{i}$ has $C M$ by $\mathbf{Z}[(1+\sqrt{-7}) / 2]$, and for $i \neq j, \mathbf{E}_{i}$ is canonically isomorphic to $\mathbf{E}_{j}$.

Proof of Proposition 4. Notice that $w_{i}=\operatorname{Tr}_{F_{i}}^{K(\mathbf{X})}(x)$, and $v_{i}=\operatorname{Tr}_{F_{i}}^{K(\mathbf{X})}(y)$, where $\operatorname{Tr}$ denotes the trace map. Since $w_{i}$ and $v_{i}$ satisfy

$$
v_{i}^{2}-3 \zeta^{4 i} v_{i} w_{i}+\zeta^{5 i} v_{i}=-w_{i}^{3}+2 \zeta^{2 i} w_{i}-3 \zeta^{3 i}
$$


they generate over $K$ a subfield of $K(\mathbf{X})$ which is contained in $F_{i}$. Since the degree of the extension $K(\mathbf{X}) / K\left(w_{i}, v_{i}\right)$ is 3, we have $K\left(w_{i}, v_{i}\right)=F_{i}$.

Notice that the morphism $\phi_{i}$ is only ramified at the points $\left(\zeta^{i} \omega, \zeta^{5 i} \omega^{2}\right)$ and $\left(\zeta^{i} \omega^{2}, \zeta^{5 i} \omega\right)$, where $\omega$ is a cubic root of 1 in $\bar{Q}$. The fibre over $\phi_{i}(P)$ is $\left\{P, \sigma_{i}(P), \sigma_{i}^{2}(P)\right\}$. Also notice that $\mathbf{E}_{i}$ has discriminant $-\zeta^{6 i} \cdot 7^{3}$, and $j$-invariant $-5^{3} \cdot 3^{3}$.

For $i \neq j, K\left(\mathbf{E}_{i}\right)$ and $K\left(\mathbf{E}_{j}\right)$ are intermediate extensions of $K(\mathbf{X}) / K(\mathbf{X})^{\langle\sigma, \varepsilon\rangle}$ (where $K(\mathbf{X})^{\langle\sigma, \varepsilon\rangle}$ is the fixed field of $\langle\sigma, \varepsilon\rangle$ ), corresponding to the conjugate subgroups $\left\langle\sigma_{i}\right\rangle$ and $\left\langle\sigma_{j}\right\rangle$. Therefore the function fields and hence the curves are isomorphic.

It remains to show that $\mathbf{E}_{i}$ has $\mathbf{C M}$ by the maximal order in $\mathbf{Q}(\sqrt{-7})$. Since the $\mathbf{E}_{i}$ 's are isomorphic it suffices to work with $i=0$. Define $e \in \operatorname{End}\left(\mathbf{E}_{0}\right)$ by

$$
e(P)=\phi_{0}(\zeta \bar{P})+\phi_{0}\left(\zeta^{2} \bar{P}\right)+\phi_{0}\left(\zeta^{4} \bar{P}\right),
$$

where $\bar{P} \in \mathbf{X}$ is such that $\phi_{0}(\bar{P})=P$. Existence of $\bar{P}$ follows by surjectivity of $\phi_{0}$, and one can easily check that $e$ is well defined, since $\zeta \sigma=\sigma \zeta^{2}$ in $\operatorname{Aut}(\mathbf{X})$. Furthermore we have

$$
\left(e^{2}+e+2\right)(P)=2 \sum_{i=1}^{7} \phi_{0}\left(\zeta^{i} \bar{P}\right)=0 .
$$

Hence by mapping the quotient $(-1+\sqrt{-7}) / 2$ to $e$ we obtain an endomorphism of $\mathbf{Z}[(1+\sqrt{-7}) / 2]$ to $\operatorname{End}\left(\mathbf{E}_{0}\right)$, which must be an isomorphism since $\mathbf{Z}[(1+\sqrt{-7}) / 2]$ is the maximal order in $\mathbf{Q}(\sqrt{-7})$. This completes the proof of Proposition 4.

The following lemma will be used in $\S 3$.

Lemma 5. With notation as above, on $\mathbf{J}_{\mathbf{X}}$ we have

$$
\operatorname{Ker}\left(\phi_{i}\right)_{*}=\operatorname{Ker}\left(1+\sigma_{i}+\sigma_{i}^{2}\right) \text {. }
$$

Furthermore, for $i \neq j, \operatorname{Ker}\left(\phi_{i}\right)_{*} \neq \operatorname{Ker}\left(\phi_{j}\right)_{*}$.

Proof of Lemma 5. If $D \in \mathbf{J}_{\mathbf{X}}$ is such that $\left(\phi_{i}\right)_{*} D=(f)$ for some $f \in K\left(\mathbf{E}_{i}\right)$, then $\left(1+\sigma_{i}+\sigma_{i}^{2}\right) D=\left(f \circ \phi_{i}\right)$; hence, on $\mathbf{J}_{\mathbf{X}}, \operatorname{Ker}\left(\phi_{i}\right)_{*} \subseteq \operatorname{Ker}\left(1+\sigma_{i}+\sigma_{i}^{2}\right)$. Conversely, if $\left(1+\sigma_{i}+\sigma_{i}^{2}\right) D=(g)$, for some $g \in K(\mathbf{X})$, then $\left(\sigma_{i} g / g\right)=0$; hence, $\sigma_{i} g=\lambda g, \lambda \in K$. Hence

$$
\left(\sigma_{i}+\sigma_{i}^{2}+1\right) g=\lambda^{3}\left(1+\sigma_{i}+\sigma_{i}^{2}\right) g .
$$

Since $K$ contains no nontrivial cubic roots of $1, \lambda=1$, that is $\sigma_{i}(g)=g$; therefore $g \in F_{i}$. Hence $g=f \circ \phi_{i}$, with $f \in K\left(\mathbf{E}_{i}\right)$. Therefore $\left(\phi_{i}\right)_{*} D=(f)$, and hence $\operatorname{Ker}\left(1+\sigma_{i}+\sigma_{i}^{2}\right) \subseteq \operatorname{Ker}\left(\phi_{i}\right)_{*}$. To prove that for $i \neq j, \operatorname{Ker}\left(\phi_{i}\right)_{*} \neq$ $\operatorname{Ker}\left(\phi_{j}\right)_{*}$, we compute $\mathbf{E}_{0}[2]$, the 2-torsion subgroup of $\mathbf{E}_{0}$. We have

$$
\mathbf{E}_{0}[2]=\{R, \bar{R}, R+\bar{R}, \mathbf{O}\},
$$

where $R=((-13+\sqrt{-7}) / 8,(31+3 \sqrt{-7}) / 16)$. (Observe that $R+\bar{R}=(1,-2) \in$ $\mathbf{E}_{0}(\mathbf{Q})$.) Since $\mathbf{D}=P+\sigma P-2 \infty_{1}$, a direct computation gives $\phi_{0}(P)=(1,-2)$; hence

$$
\left(\phi_{0}\right)_{*} \mathbf{D}=2(R+\bar{R})-20=\mathbf{0},
$$


while for $i=1$ or 2 ,

$$
\left(\phi_{i}\right)_{*} \mathbf{D}=\left(\zeta^{i}\left(\mu_{1}-1\right), \zeta^{5 i}\left(1+\mu_{1}\left(\zeta+\zeta^{2}+\zeta^{4}\right)^{2}\right)\right) \neq \mathbf{O} .
$$

(In fact one can show that $\left(\phi_{i}\right)_{*} \mathbf{D} \in \mathbf{E}_{i}[\sqrt{-7}]$ for $i \neq 0$, but we will not use this here.) Using the isomorphism of the curves $\mathbf{E}_{i}$, it follows that the kernels of the maps $\left(\phi_{i}\right)_{*}$ are pairwise distinct. This completes the proof of Lemma 5.

\section{The ACTION OF $\phi_{*}$ ON $\mathbf{J}_{\mathbf{X}}\left[\pi^{3}\right]$}

Let $R=\mathbf{Z}[\zeta] /\left(\pi^{3}\right)$. We fix a generator $\beta$ of $\mathbf{J}_{\mathbf{X}}\left[\pi^{3}\right]$ as a principal $R$ module. Then $\beta$ has exact order $\pi^{3}$ and the action of $\sigma$ on $\mathbf{J}_{\mathbf{X}}\left[\pi^{3}\right]$ is completely determined by its action on $\beta$. Since $\sigma \in \operatorname{Aut}\left(\mathbf{J}_{\mathbf{X}}\right)$, we have

$$
\sigma(\beta)=\alpha_{\sigma} \beta, \quad \alpha_{\sigma} \in R^{*} .
$$

We will now determine $\alpha_{\sigma}$. Notice that $R^{*}$ is a $\langle\sigma\rangle$ module via the action

$$
\left(\sum_{i=1}^{7} a_{i} \zeta^{i}\right)^{\sigma}=a_{i} \zeta^{4 i}, \quad a_{i} \in \mathbf{Z},
$$

and since $\sigma \zeta=\zeta^{4} \sigma$ on $\mathbf{J}_{\mathbf{X}}$, the map $\sigma \rightarrow \alpha_{\sigma}$ gives rise to a cocycle in $H^{1}\left(\langle\sigma\rangle, R^{*}\right)$. Since $\sigma^{3}=1$, we have $\beta=\sigma^{3} \beta=\alpha_{\sigma}^{1+\sigma+\sigma^{2}} \beta$, and therefore

$$
\alpha_{\sigma}^{1+\sigma+\sigma^{2}}=1
$$

in $R$. We will prove:

Lemma 6. With notation as above, $\alpha_{\sigma}=2$.

For the proof of Lemma 6 we will need Lemma 7 stated below. Consider the exact sequence of $\langle\sigma\rangle$ modules:

$$
0 \rightarrow\left(1+\pi \mathbf{Z}[\zeta] /\left(\pi^{3}\right)\right) \rightarrow R^{*} \rightarrow(\mathbf{Z} / 7 \mathbf{Z})^{*} \rightarrow 0
$$

with the action of $\sigma$ as described in (3.1). We get the long exact sequence of cohomology:

$$
\begin{aligned}
\cdots & \rightarrow H^{1}\left(\langle\sigma\rangle, 1+\pi \mathbf{Z}[\zeta] /\left(\pi^{3}\right)\right) \rightarrow H^{1}\left(\langle\sigma\rangle, R^{*}\right) \\
& \rightarrow H^{1}\left(\langle\sigma\rangle,(\mathbf{Z} / 7 \mathbf{Z})^{*}\right) \rightarrow H^{2}\left(\langle\sigma\rangle, 1+\pi \mathbf{Z}[\zeta] /\left(\pi^{3}\right)\right) \rightarrow \cdots .
\end{aligned}
$$

We will prove:

Lemma 7. Let $B=1+\pi \mathrm{Z}[\zeta] /\left(\pi^{3}\right)$. Then for $i=1$ and 2 we have $H^{i}(\langle\sigma\rangle, B)$ $=0$.

Proof of Lemma 7. Since $\langle\sigma\rangle$ is finite and cyclic, we have [8, Theorem 10.35]

$$
H^{1}(\langle\sigma\rangle, B)=B_{0} / B^{1-\sigma},
$$

while

$$
H^{2}(\langle\sigma\rangle, B)=B_{1} / B^{\left(1+\sigma+\sigma^{2}\right)},
$$

where $B_{0}=\left\{b \in B \mid b^{1+\sigma+\sigma^{2}}=1\right\}$, and $B_{1}=\left\{b \in B \mid b^{\sigma}=b\right\}$. Also it follows from (3.1) that in $R$ we have

$$
\pi^{\sigma} \equiv-\pi^{2}+4 \pi \bmod \pi^{3}, \quad \pi^{\sigma^{2}} \equiv \pi^{2}+2 \pi \bmod \pi^{3} .
$$


A quick computation yields that $B_{0}=B^{1-\sigma}=B$, and that $B_{1}=B^{1+\sigma+\sigma^{2}}=\{1\}$. So Lemma 7 follows.

Proof of Lemma 6. It follows from Lemma 7 that the homomorphism $H^{1}\left(\langle\sigma\rangle, R^{*}\right) \rightarrow H^{1}\left(\langle\sigma\rangle,(\mathbf{Z} / 7 \mathbf{Z})^{*}\right)$ is an isomorphism. Hence $\left.\alpha_{\sigma} \in(\mathbf{Z} / 7 \mathbf{Z})^{*}\right)$ and (3.2) gives the result that $\alpha_{\sigma}^{3}=1$, hence $\alpha_{\sigma}=1,2$, or 4 . We will next rule out the cases $\alpha_{\sigma}=1$ and $\alpha_{\sigma}=4$.

We have

$$
\mathbf{J}_{\mathbf{X}}\left[\pi^{3}\right]=\left\{\left(a+b \pi+c \pi^{2}\right) \boldsymbol{\beta} \mid a, b, c \in(\mathbf{Z} / 7 \mathbf{Z})\right\} .
$$

Using Lemma 5 and (3.3) we can compute $\operatorname{Ker}\left(\phi_{i}\right)_{*}$ for $i=0$, 1 , or 2 .

If $\alpha_{\sigma}=1$, we obtain

$$
\left(1+\sigma+\sigma_{i}^{2}\right)\left(a+b \pi+c \pi^{2}\right) \beta=3 a \beta \text { for } i=0,1,2 .
$$

But this implies that for $i=0,1$, or 2 , we have

$$
\operatorname{Ker}\left(\phi_{i}\right)_{*}=\left\{\left(b \pi+c \pi^{2}\right) \beta \mid a, c \in(\mathbf{Z} / 7 \mathbf{Z})\right\}=\mathbf{J}_{\mathbf{X}}\left[\pi^{2}\right],
$$

which contradicts Lemma 5.

If $\alpha_{\sigma}=4$, we obtain

$$
\left(1+\sigma+\sigma^{2}\right)\left(a+b \pi+c \pi^{2}\right) \beta=(3 c-2 b) \pi^{2} \beta ;
$$

hence $\mathbf{J}_{\mathbf{X}}[\pi]=\left\{\left(c \pi^{2}\right) \beta \mid c \in(\mathbf{Z} / 7 \mathbf{Z})\right\}$ is not contained in $\operatorname{Ker}\left(\phi_{0}\right)_{*}$. This is a contradiction, because $\left(\phi_{0}\right)_{*}\left(\infty_{2}-\infty_{1}\right)=\mathbf{O}$, and as noted in the introduction, $\infty_{2}-\infty_{1}$ generates the $\pi$-torsion on $\mathbf{J}_{\mathbf{X}}$. Hence $\alpha_{\sigma}=2$.

Next we will prove:

Lemma 8. With notation as above, for $i=0,1,2$ we have $\operatorname{Ker}\left(\phi_{i}\right)_{*} \cap \mathbf{J}_{\mathbf{X}}\left[\pi^{2}\right]=$ $\mathbf{J}_{\mathbf{X}}[\pi]$.

Proof of Lemma 8. Using Lemmas 5 and 6 along with (3.3), a direct computation gives

$$
\operatorname{Ker}\left(\phi_{i}\right)_{*} \cap \mathbf{J}_{\mathbf{X}}\left[\pi^{3}\right]= \begin{cases}\left\{\left(a+c \pi^{2}\right) \beta \mid a, c \in(\mathbf{Z} / 7 \mathbf{Z})\right\} & \text { if } i=0, \\ \left\{\left(a-a \pi+c \pi^{2}\right) \beta \mid a, c \in(\mathbf{Z} / 7 \mathbf{Z})\right\} & \text { if } i=1, \\ \left\{\left(a+2 a \pi+c \pi^{2}\right) \beta \mid a, c \in(\mathbf{Z} / 7 \mathbf{Z})\right\} & \text { if } i=2 .\end{cases}
$$

Then Lemma 8 follows.

Next we will show:

Lemma 9. With notation as above we have

$$
\operatorname{Ker}\left(\phi_{0}\right)_{*} \cap \operatorname{Ker}\left(\phi_{1}\right)_{*} \cap \operatorname{Ker}\left(\phi_{2}\right)_{*}=\mathbf{J}_{\mathbf{X}}[\pi]
$$

Proof of Lemma 9. Suppose that for $i=0,1$, or $2, D \in \operatorname{Ker}\left(\phi_{i}\right)_{*}$. Then by Lemma $5\left(1+\sigma_{i}+\sigma_{i}^{2}\right) D=0$. Using the relations

$$
1+\sigma_{i}+\sigma_{i}^{2}=1+\zeta^{4 i} \sigma+\zeta^{2 i} \sigma^{2}, \quad i=1,2,
$$

it is easy to check that in $\operatorname{End}\left(\mathbf{J}_{\mathbf{X}}\right)$ we have

$(\zeta-1)^{2}(\zeta+1) \zeta\left(\zeta^{2}+\zeta+1\right) \sigma=\zeta^{6}\left(1+\sigma+\sigma^{2}\right)-\left(1+\zeta^{6}\right)\left(1+\sigma_{1}+\sigma_{1}^{2}\right)+\left(1+\sigma_{2}+\sigma_{2}^{2}\right)$.

It follows that $(\zeta-1)^{2} D=0$, since $\zeta+1, \zeta, \zeta^{2}+\zeta+1$, and $\sigma$ are all units in $\operatorname{End}\left(\mathbf{J}_{\mathbf{X}}\right)$. Hence $D \in\left(\mathbf{J}_{\mathbf{X}}\left[\pi^{2}\right]\right)$. Then by Lemma 8 we have that $D \in \mathbf{J}_{\mathbf{X}}[\pi]$. The other inclusion is obvious. 


\section{THE PROOF OF THEOREMS 1 AND 2}

Recall that $\mathbf{D}=P+\sigma P-2 \infty_{1}$.

Proof of Lemma 1. We will first show that D has exact order $\pi^{3}$. Using Lemmas 7 and 8 , it suffices to show that

(1) $\mathbf{D} \in \mathbf{J}_{\mathbf{X}}\left[\pi^{3}\right]$,

(2) $\mathbf{D} \in \operatorname{Ker}\left(\phi_{0}\right)_{*}$, and

(3) $\mathbf{D} \notin \operatorname{Ker}\left(\phi_{1}\right)_{*}$.

To prove (1), consider $f \in K(\mathbf{X})$ given by

$$
f=\zeta \mu_{2} x^{2}+y^{2}+\zeta^{3} x y+\frac{\zeta^{4}}{\mu_{3}} y+\frac{\mu_{2}}{\zeta} x+\frac{\zeta^{5}}{\mu_{3}} \text {. }
$$

Then the divisor of $f$ is

$$
(f)=\left(\left(\zeta+\zeta^{2}+\zeta^{4}\right)(P+\sigma P)\right)+P+\sigma^{2} P-6 \infty_{2}-2 \infty_{1} .
$$

Since $P, \sigma P$, and $\sigma^{2} P$ are fixed under complex conjugation we have

$$
\left(\frac{f}{\bar{f}}\right)=\left(\left(\zeta+\zeta^{2}+\zeta^{4}\right)-\left(\zeta^{3}+\zeta^{5}+\zeta^{6}\right)\right) \mathbf{D},
$$

where $\bar{f}$ denotes the complex conjugate of $f$. That is, we have $\left(\pi^{3}\right) \mathbf{D}=0$, because

$$
\left(\zeta+\zeta^{2}+\zeta^{4}\right)-\left(\zeta^{3}+\zeta^{5}+\zeta^{6}\right)=(\zeta-1)^{3}(\zeta+1)^{2}\left(\zeta^{2}+1\right)
$$

and $\zeta+1, \zeta^{2}+1$ are units in $Z[\zeta]$.

Notice also that (2) and (3) follow from (2.3) and (2.4).

To complete the proof of Theorem 1 it remains to show that $\mathbf{J}_{\mathbf{X}, \mathbf{w}}=\mathbf{J}_{\mathbf{X}}\left[2 \pi^{3}\right]$. Notice that

$$
\mathbf{D}_{0}=P \nmid \sigma P+\sigma^{2} P-\infty_{1}-\infty_{2}-\infty_{3} \in \mathbf{J}_{\mathbf{X}}(\mathbf{Q})
$$

has exact order 2 since $\mathbf{D}_{0}=\left(\phi_{0}\right)_{*}^{-1}((2,-1)-\mathbf{O})$. But then the points $\mathbf{D}_{i}=$ $\zeta^{i} \mathbf{D}_{0}, i=0,1, \ldots, 5$ are independent [4, Lemma 1.3] and hence

$$
(\mathbf{Z} / 2 \mathbf{Z})^{6} \subseteq \mathbf{J}_{\mathbf{X}, \mathbf{w}}
$$

Therefore $\mathbf{J}_{\mathbf{X}}\left[2 \pi^{3}\right] \subseteq \mathbf{J}_{\mathbf{X}}, \mathbf{w}$. Conversely, since $P-\infty_{1}=\mathbf{D}_{0}-\mathbf{D}+\left(\infty_{2}+\infty_{3}-\right.$ $\left.2 \infty_{1}\right)$, it follows that $\left(P-\infty_{1}\right) \in \mathbf{J}_{\mathbf{X}}\left[2 \pi^{3}\right]$. Therefore $\mathbf{J}_{\mathbf{X}}, \mathbf{w} \subseteq \mathbf{J}_{\mathbf{X}}\left[2 \pi^{3}\right]$. This completes the proof of Theorem 1 .

Proof of Theorem 2. Consider the isogeny

$$
\phi: \mathbf{J}_{\mathbf{X}} \rightarrow \mathbf{E}_{0} \times \mathbf{E}_{1} \times \mathbf{E}_{2}
$$

studied in $\S 2$. We have from Lemma 9 that $\operatorname{Ker}(\phi)_{*}=\mathbf{J}_{\mathbf{X}}[\pi]$. Hence we may define an isomorphism $l_{\phi}$ as follows: Given $D \in \mathbf{J}_{\mathbf{X}}$, let $\bar{D}$ be such that $\pi \bar{D}=D$. Since $\pi$ is a surjective endomorphism, such a $\bar{D}$ always exists. Then define $l_{\phi}(D)=\phi_{*}(\bar{D})$. It is easy to check that $l_{\phi}$ is well defined and a birational isomorphism, hence an isomorphism of abelian varieties. This completes the proof of Theorem 2.

\section{ACKNOWLEDGMENTS}

I would like to thank my thesis advisor Robert Coleman for suggesting the problem to me, and for teaching and encouraging me throughout this study. 


\section{REFERENCES}

1. R. Coleman, Torsion points on curves and p-adic abelian integrals, Ann. of Math. (2) 121 (1985), 111-165.

2. Torsion points on Abelian etale coverings of $P^{1}-\{0,1, \infty\}$, Trans. Amer. Math. Soc. 311 (1989), 185-208.

3. R. Greenberg, On the Jacobian variety of some algebraic curves, Compositio Math. $\mathbf{4 2}$ (1981), 345-359.

4. B. Gross and D. Rohrlich, Some results on the Mordell-Weil group of the Jacobian of the Fermat curve, Invent. Math. 44 (1978), 201-224.

5. F. Klein, Uber die Transformation siebenter Ordhang der elliptischen Functionen, Gesammelte Math. Abhandlungen III, vol. 84, Springer, Berlin, 1923.

6. N. Koblitz and D. Rohrlich, Simple factors in the Jacobian of the Fermat curve, Canad. J. Math. 20 (1978), 1183-1205.

7. S. Lang, Complex multiplication, Springer-Verlag, New York, 1983.

8. J. Rotman, An introduction to homological algebra, Academic Press, New York, 1979.

9. D. Springer, Introduction to Riemann surfaces, Addison Wesley, Reading, MA, 1957.

Department of Mathematics, Diablo Valley College, Pleasant Hill, California 94523 